\title{
Corrigendum
}

\section{Corrigendum to "Multimedia Archives: New Digital Filters to Correct Equalization Errors on Digitized Audio Tapes"}

\author{
Niccolò Pretto (iD, ${ }_{1}^{1}$ Edoardo Micheloni $\left(\mathbb{D},{ }^{1}\right.$ Anthony Chmiel $\mathbb{D}^{\mathrm{D}},{ }^{2}$ Nadir Dalla Pozza $\left(\mathbb{D},{ }^{1}\right.$ \\ Dario Marinello, ${ }^{1}$ Emery Schubert $\mathbb{D}^{0},{ }^{3}$ and Sergio Canazza ${ }^{1}{ }^{1}$ \\ ${ }^{1}$ Department of Information Engineering, University of Padova, Padova, Italy \\ ${ }^{2}$ The MARCS Institute for Brain, Behaviour and Development, Western Sydney University, Sydney, Australia \\ ${ }^{3}$ Empirical Musicology Laboratory, University of New South Wales, Sydney, Australia
}

Correspondence should be addressed to Niccolò Pretto; niccolo.pretto@dei.unipd.it

Received 19 October 2021; Accepted 19 October 2021; Published 13 November 2021

Copyright (C) 2021 Niccolò Pretto et al. This is an open access article distributed under the Creative Commons Attribution License, which permits unrestricted use, distribution, and reproduction in any medium, provided the original work is properly cited.

In the article titled "Multimedia Archives: New Digital Filters to Correct Equalization Errors on Digitized Audio Tapes" [1], the authors identified an error in the presentation of equation (1) and its explanation with regard to time's unit which was expressed in microseconds in Section 2.2. Hence, corrected equation (1) and its corresponding explanation are found as follows:

$$
N(d B)=10 \log _{10}\left(1+4 \pi^{2} f^{2} t_{2}^{2}\right)-10 \log _{10}\left(1+\frac{1}{4 \pi^{2} f^{2} t_{1}^{2}}\right)
$$

where $f$ is the frequency in $\mathrm{Hz}$ and $t_{1}$ and $t_{2}$ are the time constants in seconds (derived from [11]).

In addition, the affiliation for Dr. Sergio Canazza is corrected as given above.

The authors and the editorial board agree to the publication of the corrigendum.

\section{References}

[1] N. Pretto, E. Micheloni, C. Anthony et al., "Multimedia archives: new digital filters to correct equalization errors on digitized audio tapes," Advances in Multimedia, vol. 2021, Article ID 5410218, 11 pages, 2021. 